\title{
Evaluación de la Actividad de los Movimientos en Masa de Cachi Alto-Pujilí, Ecuador Mediante Monitoreo Instrumental de Bajo Costo
}

\author{
Pilatasig, Luis $^{1, *(D)}$; Bustillos, Jorge ${ }^{1}$; Jácome, Fabián $^{1}$ (iD) Mariño, David $^{1}$ (iD \\ ${ }^{\text {I}}$ Facultad de Ingeniería en Geología, Minas, Petróleos y Ambiental, Universidad Central del Ecuador, Ciudadela Universitaria, \\ Quito, Ecuador
}

\begin{abstract}
Resumen: La zona de Cachi Alto está ubicada en la serranía ecuatoriana, formando parte de las estribaciones orientales bajas de la Cordillera Occidental, aproximadamente a $90 \mathrm{Km}$ al suroeste de Quito. El área forma parte de una extensa región donde ocurren procesos geodinámicos externos o movimientos en masa, tanto activos como inactivos de dimensiones variables. El objetivo del estudio es evaluar el estado de la actividad de los movimientos en masa, incluyendo la tendencia o dirección de movimiento de los materiales, datos con los cuales se propone también la tipología de los procesos geodinámicos. Esta investigación presenta los resultados del monitoreo instrumental realizado mediante extensómetros artesanales y estación total, combinado con cartografía de deformaciones superficiales de la corona de un deslizamiento antiguo. Las magnitudes y tendencias de los movimientos varían entre $10 \mathrm{~mm}$ y $1302 \mathrm{~mm}$ hacia el norte y sur y los resultados del monitoreo establecen desplazamientos en varias direcciones en la componente horizontal, además de asentamientos y levantamientos en la componente vertical en varias zonas ubicadas en la parte superior del deslizamiento antiguo. Con base en los resultados obtenidos, los movimientos en masa son catalogados como reactivados y toda la zona puede interpretarse como inestable. Finalmente, considerando la dinámica existente, se concluye que los procesos geodinámicos dentro del área no tienen relación con deslizamientos clásicos, razón por la cual las deformaciones superficiales posiblemente están relacionadas con procesos de reacomodamiento de bloques en una zona afectada por deformación gravitacional profunda en laderas.
\end{abstract}

Palabras clave: Movimientos en masa, monitoreo, deformación superficial, deformación gravitacional profunda, Cordillera Occidental.

\section{Evaluation of the Activity of the Mass Movements of Cachi Alto- Pujilí, Ecuador through Low-Cost Instrument Monitoring}

\begin{abstract}
The Cachi Alto zone is located in the Ecuadorian mountain range, as part of the Eastern foothills of the Western Cordillera, approximately $90 \mathrm{Km}$ to the southwest of Quito. The area is part of an extensive region where many external geodynamic processes or mass movements occur, both active and inactive of variable magnitudes. The aim of the study is to assess the state of the activity of mass movements, including the trend or direction of movement of materials, data with which the type of geodynamic processes is also proposed. This research shows the results of instrumental monitoring performed through handmade extensometers and total station theodolite, combined with surface deformation mapping of the crown of the old landslide Cachi Alto. The magnitudes of the displacements and trends vary between $10 \mathrm{~mm}$ and $1302 \mathrm{~mm}$ to the north and to the south. The monitoring results establish movements in various directions in the horizontal component, as well as settlements and uplifts in the vertical component in various zones located in the upper part of the ancient landslide. Taking into account the obtained results, the mass movements are classified as reactivated and the entire area can be interpreted as unstable. Finally, considering the existing dynamics, it is possible to conclude that geodynamic processes into the area are not related to classical landslides, which is why surface deformations are possibly related to block rearrangement processes in an area affected by deep seated gravitational slope deformation.
\end{abstract}

Keywords: Mass movements, monitoring, surface deformation, deep seated gravitational deformation, Western Cordillera.

\section{INTRODUCCIÓN}

La zona de Cachi Alto está ubicada en la serranía ecuatoriana, formando parte de las estribaciones orientales bajas de la Cordillera Occidental, aproximadamente a 90 $\mathrm{km}$ al suroeste de la ciudad de Quito y $10 \mathrm{Km}$ al suroeste de la población de Pujilí. El área forma parte de una extensa región muy susceptible a la ocurrencia de movimientos en 
masa de diversa tipología. Los principales procesos reportados son tipo deslizamientos de variada magnitud. Los estados de actividad de los mismos varían entre activos e inactivos. Los datos cartográficos cualitativos de uno de estos movimientos en masa denominado Cachi Alto, indican que es un deslizamiento antiguo o inactivo (Burga, 2019), a pesar que en la zona sur del escarpe principal ocurren deformaciones superficiales como escarpes y grietas asociadas a movimientos en masa, sin datos cualitativos y cuantitativos sobre la tipología y dinámica de las mismas. Este estudio tiene como alcance establecer las características de las laderas de Cachi Alto-Pujilí, siendo el objetivo principal evaluar el estado y la distribución de la actividad de los movimientos en masa, incluyendo la tendencia o dirección de movimiento de los materiales, datos con los cuales se propone también la tipología de los procesos geodinámicos. El presente trabajo, presenta los resultados del monitoreo mediante extensómetros artesanales y estación total (monitoreo geodésico), incluyendo información de deformaciones superficiales de la zona sur del deslizamiento antiguo, así como del escarpe principal. El análisis de los datos y la comparación de los resultados obtenidos con dos técnicas de monitoreo, incluyendo la información de la deformación superficial reciente, permite conocer la magnitud y tendencia de los movimientos de los sitios donde esta instalados los instrumentos, con lo cual se presenta una hipótesis sobre el origen, la relación con otros procesos geológicos y la tipología de los movimientos en masa.

\section{CONTEXTO MORFOLÓGICO Y GEOLÓGICO}

El área de estudio está localizada en las estribaciones orientales bajas de la Cordillera Occidental de los Andes

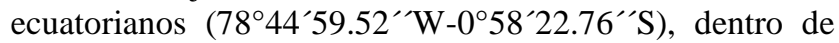
una región donde la altitud varía de 3000 a 3500 m.s.n.m. (Figura 1). El relieve es dominado por colinas con pendiente variable, de horizontal a inclinada $\left( \pm 20^{\circ}\right)$ en las zonas altas y moderadamente inclinada $\left( \pm 50^{\circ}\right)$ a vertical en los márgenes de los drenajes y en los escarpes de los movimientos en masa. La zona forma parte de la cuenca media a baja del río Patoa. El principal drenaje que atraviesa el área de estudio constituye una quebrada intermitente, cuyo valle es usado para la agricultura y ganadería de subsistencia.

La zona está constituida por rocas volcánicas y volcanoclásticas, las cuales están cubiertas por depósitos de naturaleza volcánica (Figura 2). Las rocas volcánicas y volcanoclásticas están incluidas por Burga (2019) en la Unidad Río Cala de edad Cretácico (Boland, et al. 2019) y Grupo Zumbagua (Hughes, et al., 1998), respectivamente. Los depósitos de naturaleza volcánica incluyen una capa de cangahua de hasta \pm 20 metros de espesor incluyendo capas centimétricas de lapilli, las cuales en la zona sur están cubiertas por una delgada capa de suelo de hasta \pm 1 metro de espesor y en la zona norte por un depósito coluvial antiguo (Burga, 2019) de aproximadamente 10 metros de espesor. Este último está relacionado con el movimiento en masa de Cachi Alto. Todas las secuencias litológicas están deformadas y tectonizadas, con excepción del depósito coluvial.

La deformación y el tectonismo están relacionados con tres sistemas de fallamiento que atraviesa la región (Figura 2). Un sistema de fallamiento tipo inverso de rumbo $\mathrm{N}-\mathrm{S}$ buzando al oeste, relacionado con el sistema de fallas Pallatanga-Pujilí-Calacalí (Mc Court, et al., 1997); otro sistema tipo inverso de rumbo NE-SW buzando al SE, posiblemente relacionado con la falla Tambillo (Mc Court, et al., 1997) y un tercer sistema tipo transcurrente de rumbo E-W.

Los movimientos en masa establecidos en la región mediante información sismológica datan de 1962 a partir de dos sismos ocurridos en la zona (Egred, informe inédito no publicado). En 1995 (un año antes de sismo de Pujilí de 1996), otro movimiento en masa complejo denominado Cuturiví de enorme magnitud $\left( \pm 1,8 \mathrm{~km}^{2}\right)$ fue reportado al sur y sur este de Cachi Alto (Paladines, et al, informe inédito no publicado). Considerando la clasificación de Cruden y Varnes (1996) y mediante información cartográfica Pilatasig, et al. (2018) y Burga (2019) determinan que en la zona de Cachi Alto ocurren tres deslizamientos, dos de tipo traslacional y un deslizamiento antiguo posiblemente también de tipo traslacional (Figuras 2a y 2b).

Los deslizamientos son de pequeña $\left( \pm 0,003 \mathrm{~km}^{2}\right)$, mediana $\left( \pm 0,017 \mathrm{~km}^{2}\right)$ y gran magnitud $\left( \pm 0,12 \mathrm{~km}^{2}\right)$.

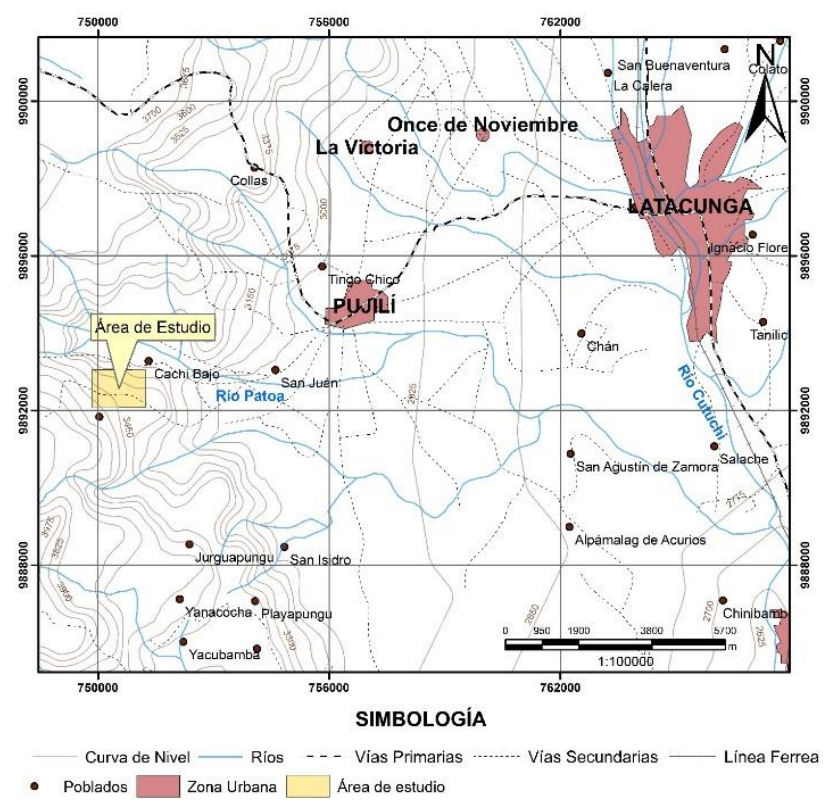

Figura 1. Esquema topográfico de las estribaciones orientales bajas de la cordillera Occidental y la zona occidental del Valle Interandino, incluyendo la localización del área de estudio, ubicada a unos $7 \mathrm{Km}$. al sur-oeste de Pujilí

En la zona sur del escarpe principal del movimiento en masa de Cachi Alto o corona del deslizamiento antiguo, ocurren varias estructuras que incluyen escarpes con rumbos $\mathrm{NE}$ SW, NW-SE y E-W además de varias fracturas o grietas, algunas de las cuales están rellenas con suelo (Burga, 2019). 
El origen y relación de estas estructuras con el fallamiento o los movimientos en masa aún es incierta.

Con respecto al tipo de movimiento en masa, además de los definidos por Cruden y Varnes (1996), Hutchinson (1988) determina la ocurrencia de una variedad de procesos que se definen como deformaciones de ladera o deformaciones gravitacionales profundas (Deep-seated gravitational slope deformation, término en inglés).

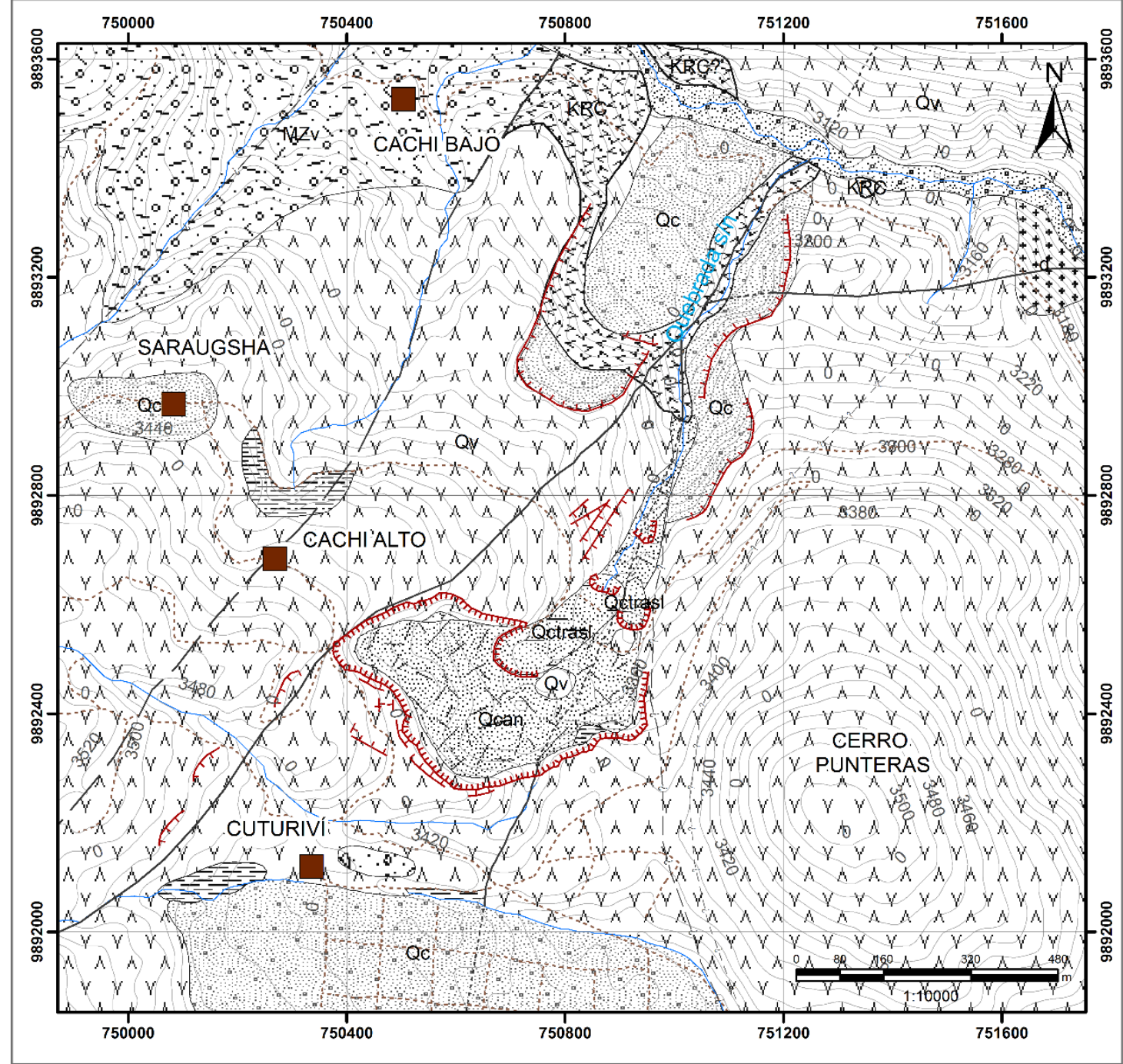

\section{LEYENDA GEOLÓGICA}

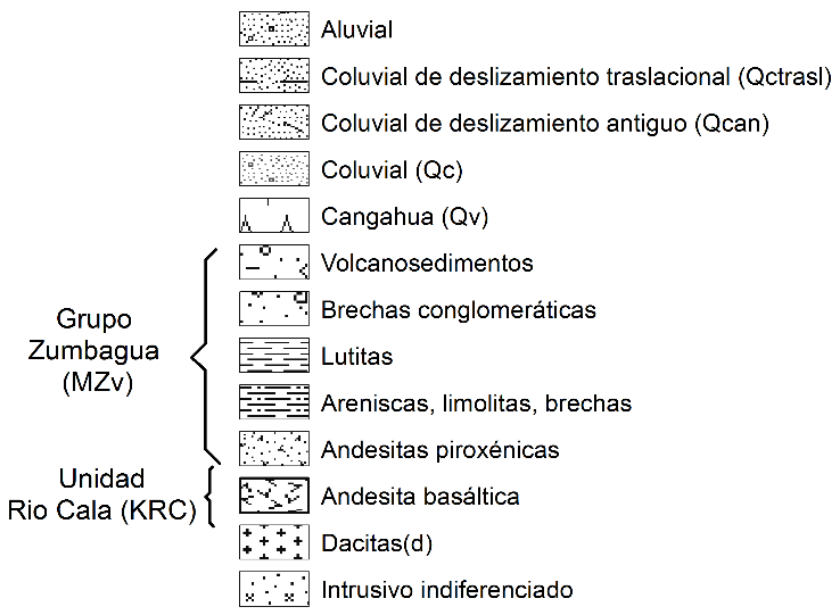

SIMBOLOGÍA GEOLÓGICA

TाT Escarpe

-....-. Falla cubierta

-? - - Falla inferida

- Falla observada

- - Lineamiento

Contacto inferido

SIMBOLOGÍA TOPOGRÁFICA

Poblados

Curvas de nivel

....... Vías

$\frown$ Quebradas

Figura 2a. Esquema geológico-estructural de la zona de Cachi Alto y su área de influencia, incluyendo escarpes asociados a los deslizamientos principales (Modificado de Burga, 2019) 


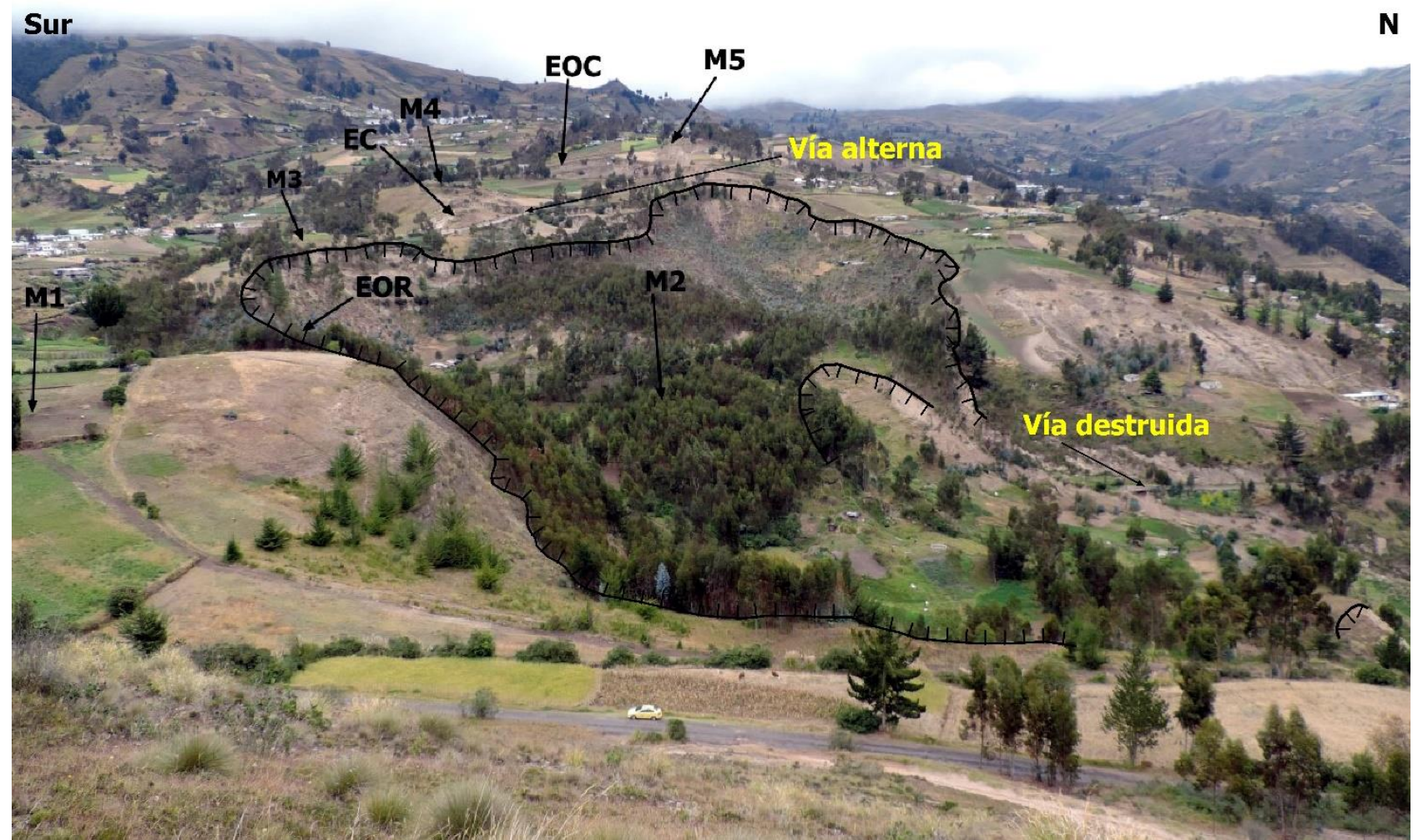

Figura 2b. Imagen mostrando el deslizamiento antiguo de Cachi Alto y los dos deslizamientos traslacionales, incluyendo la ubicación de los instrumentos de monitoreo ubicados en la corona del deslizamiento. El Mojón 2 (M2) y Extensómetro oriental (EOR) están ubicados en el cuerpo del deslizamiento y en el escarpe principal

Estos tipos de movimientos en masa presentan rasgos de deformación sin desarrollo de superficie de rotura definidas y con baja magnitud de velocidad y desplazamiento, además algunas deformaciones de ladera deben ser consideradas como precursoras de deslizamientos en gran escala (PMA: GCA, 2007).

La relación entre el fallamiento activo y regiones de alto relieve con movimientos en masa catalogados como deformaciones gravitacionales han sido determinados en varias regiones montañosas como Los Andes de Mérida, Venezuela (Audemard, et al., 2010) y Tien Shan Mountains, Asia Central (Teshebaeva, et al., 2019). Estos procesos descritos como deformaciones de laderas (slope deformation) o deformaciones gravitacionales profundas también son reportados por Riemer et al. (1988) en la Cordillera Real de los Andes.

Considerando la terminología de Cruden y Varnes (1996), el estado de actividad de los dos deslizamientos de pequeña y mediana magnitud son catalogados como activos, mientras del deslizamiento de gran magnitud es inactivo o antiguo (Burga, 2019). Los dos deslizamientos activos son condicionados por la actividad antrópica a la que se suma la morfología y la pluviosidad, mientras el deslizamiento antiguo es condicionado por actividad neotectónica (Burga, 2019).

\section{CONTEXTO METODOLÓGICO}

El primer paso de la investigación incluyó el diseño y construcción de los extensómetros con base en el modelo propuesto por León et. al (2007) y los mojones metálicos (Figura 3). La construcción de los equipos-instrumentos fue realizada por Pérez (2019).

Los extensómetros fueron utilizados para medir las dimensiones de las grietas. Los mojones metálicos en conjunto con la Estación Total permitieron establecer datos geodésicos absolutos (UTM X, UTM Y y Altura), con los cuales se definió el movimiento en los componentes horizontal (Este-Oeste o UTM X y Norte-Sur o UTM Y) y vertical (levantamiento o hundimiento). Cada extensómetro consiste de 2 tubos, 1 cable, 2 poleas, 1 regla metálica, 3 sujetadores del cable, 1 ceja de medición y 1 pesa.

El ensamblaje de los componentes forma dos postes denominados Tubos Fijo y Móvil (Figura 3a). El Fijo incluye una regla metálica, polea, sujetador metálico, ceja de medición y pesa. El Móvil incluye polea y sujetador metálico. 
Por otra parte, cada mojón consiste de un tubo redondo, un perno compatible con la rosca del prisma óptico y placa metálica con 2 varillas de hierro corrugado como base (Figura 3b). La Estación Total es marca Trimble modelo 2600 y tiene una precisión o fiabilidad de $1 \mathrm{~mm}+1 \mathrm{ppm}$.

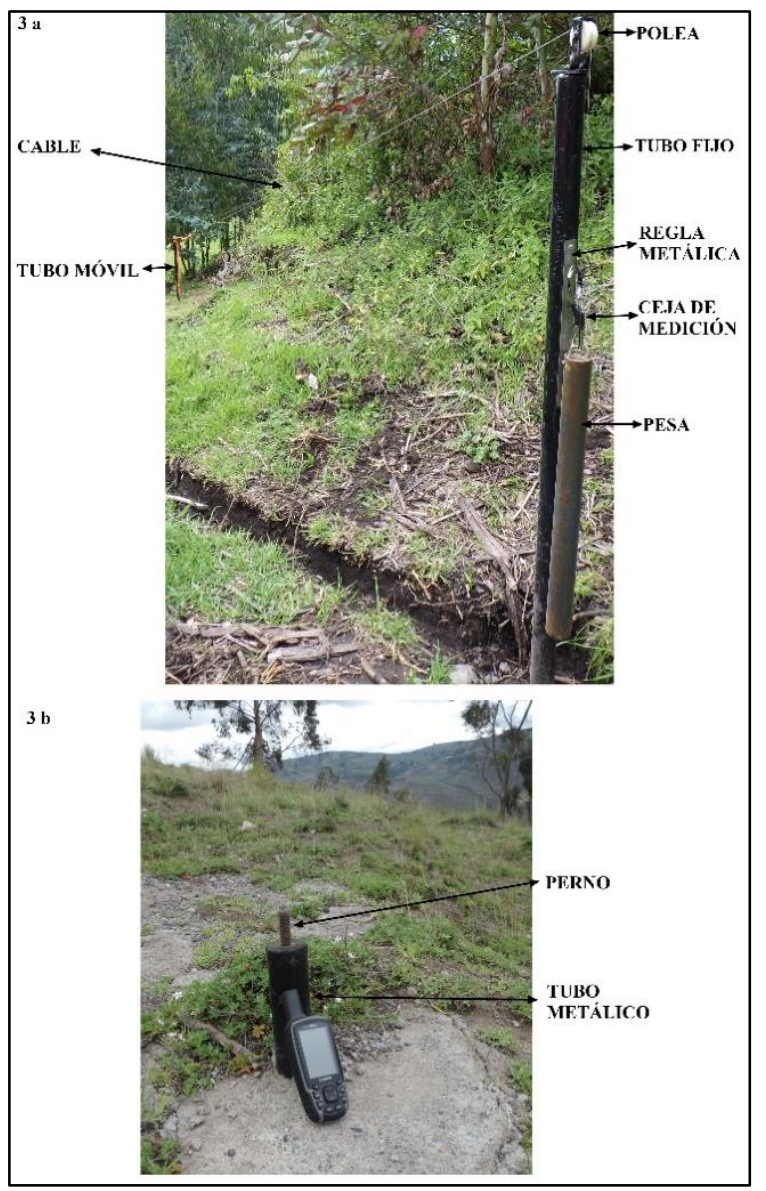

Figura 3. Extensómetro ensamblado e instalado, incluye los tubos implantados en la zona activa o inestable (Tubo móvil) y en la zona estable o sin movimiento (Tubo fijo), además de los componentes principales (3a).

Mojón metálico instalado en el cerro Punteras denominado Estación Base,

incluyendo el perno para instalar el prisma óptico y parte del tubo empotrado en un hoyo relleno de hormigón (3b)

El siguiente paso fue la instalación de los extensómetros y mojones, incluyendo el Mojón Base en los sitios previamente definidos (Figura 4). Las posiciones geográficas de los sitios fueron establecidas con un GPS GARMIN 64S. El mojón base está ubicado en el Cerro Punteras en el punto de coordenadas 751 237-9 892235 (Sistema WGS84).

La sujeción de los tubos se realizó con concreto y fragmentos de rocas. El ensamblaje de los extensómetros se ejecutó en el sitio de emplazamiento. La investigación de campo ejecutada desde octubre de 2019 hasta marzo de 2020, incluyó el registro de datos de los extensómetros y Estación Total durante diez días y dos campañas de campo, respectivamente, además del cartografiado y levantamiento de información de deformaciones superficiales.

La información constituye la base con la cual se define el área total de la zona inestable, el estado de la actividad de la corona del deslizamiento antiguo y la tendencia o dirección de los movimientos en masa.

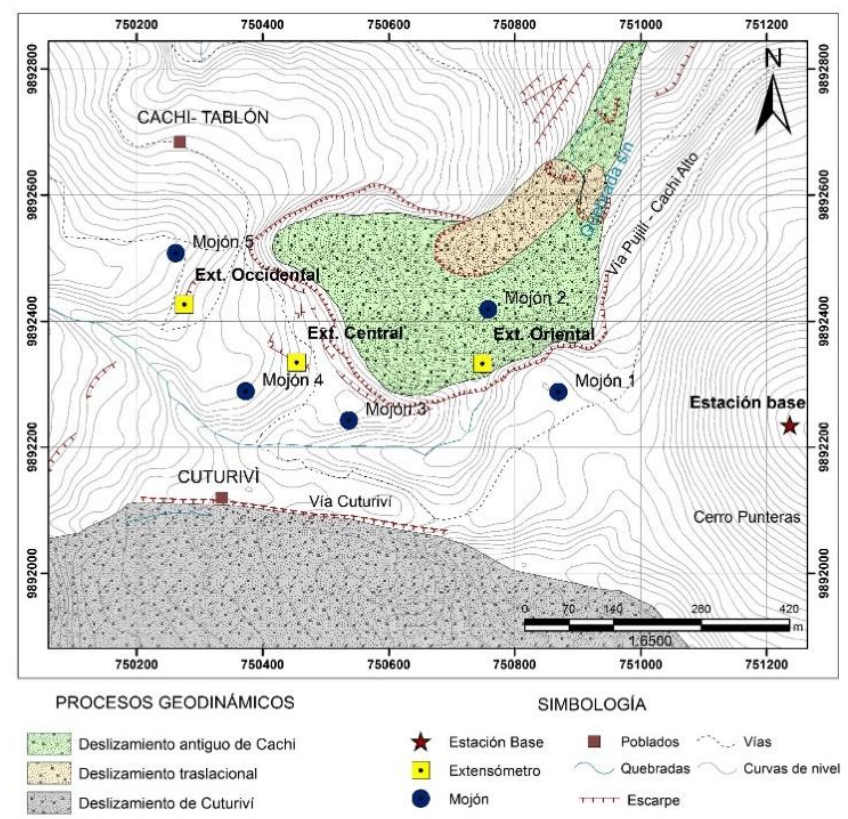

Figura 4. Esquema de los deslizamientos activos ubicado en la zona norte relacionados con quebrada s/n y el deslizamiento antiguo de Cachi Alto, incluyendo la ubicación de los extensómetros artesanales y los mojones

En cada uno de los lugares se excavaron hoyos de $100 \mathrm{~cm}$ de profundidad y $50 \mathrm{~cm}$ de diámetro. Todos los hoyos están excavados en cangahua, con excepción de los dos hoyos del Extensómetro Oriental, los cuales están excavados en depósito coluvio-aluvional.

\section{ESTADO Y DISTRIBUCIÓN DE LA ACTIVIDAD DE LOS MOVIMIENTOS EN MASA DE CACHI ALTO- PUJILÍ}

La gran cantidad de movimientos en masa en la zona de Cachi Alto y su área de influencia sugieren que la zona es susceptible a la ocurrencia de movimientos en masa de diversa tipología, magnitud y estado de actividad (Figura 2). El desplazamiento de los cuerpos de los dos deslizamientos activos que ocurren en la zona de Cachi es variable desde $\pm 1 \mathrm{~m}$ hasta $\pm 15 \mathrm{~m}$ en un periodo de 6 meses, este último determinado en base al desplazamiento de una parte de la vía antigua Pujilí-Cachi Alto que atraviesa por la zona norte del deslizamiento antiguo de Cachi Alto.

En los deslizamientos catalogados como antiguos o inactivos donde ocurren deformaciones superficiales recientes como fracturas o grietas y escapes, la dinámica es imperceptible a los sentidos humanos. La determinación del estado de la actividad en esta zona está basada en datos cuantitativos registrados en los instrumentos y cualitativos levantados en las zonas donde están instalados los instrumentos y en el área de influencia de los mismos.

Las magnitudes de las grietas formadas en las zonas suroriental, sur central y suroccidental del movimiento en 
masa en base del desplazamiento de los tubos móviles son variables (Tabla 1).

En el extensómetro oriental con eje predominante norte-sur entre el 24 de octubre de 2019 y el 17 de diciembre de 2020 alcanzó hasta 79,6 cms. En el extensómetro central con eje predominante norte-sur entre el 24 de octubre de 2019 y el 10 de marzo de 2021 alcanzó hasta $1 \mathrm{~cm}$. En el extensómetro occidental con eje predominante este-oeste entre el 24 de octubre de 2019 y el 10 de marzo de 2021 alcanzó hasta $8 \mathrm{~cm}$.

Los desplazamientos de los tubos móviles en los extensómetro oriental y occidental son prácticamente constantes (Figuras 5a y 5c), mientras en el extensómetro central el desplazamiento es muy variable, así entre el 24 de octubre de 2019 hasta el 13 de noviembre del mismo año no presenta movimiento y desde 9 de enero de 2020 hasta el 10 de marzo de 2020 el movimiento es anómalo (Figura 5b).

Los desplazamientos, con base en los datos geodésicos (Tabla 2), varían desde 0,002 a 1,302 metros (Tabla 3). Las direcciones o tendencias de los desplazamientos de los mojones en el componente horizontal son muy variables y tienden al NW, NE, SW y SE (Figuras 6a, 6b, 6c, 7d y 6e). En el componente vertical los desplazamientos de los mojones son mayormente asentamientos (Figuras 7a, 7b, 7d y 7e), con excepción del mojón 3 donde ocurre levantamiento (Figura $7 \mathrm{c})$.

Tabla 1. Valores en la regla de medición y de los desplazamientos en los extensómetros oriental, central y occidental

\begin{tabular}{|c|c|c|c|c|}
\hline EXTENSÓMETRO & FECHA & $\begin{array}{c}\text { VALOR EN } \\
\text { REGLA DE } \\
\text { MEDICIÓN (CM) }\end{array}$ & $\begin{array}{c}\text { VALOR DE } \\
\text { DESPLAZAMIENTO } \\
\text { ACUMULADO }(\mathrm{CM})\end{array}$ & OBSERVACIÓN \\
\hline \multirow{10}{*}{ ORIENTAL } & 24/10/2019 & 29,0 & 0,0 & Instalación-ensamblaje (Inicio de etapa 1) \\
\hline & $3 / 11 / 2019$ & 22,2 & 6,8 & Lectura 08:35 h, después de sismo cercano \\
\hline & $3 / 11 / 2019$ & 21,5 & 7,5 & Lectura 11:35 h, después de sismo cercano \\
\hline & $9 / 11 / 2019$ & 17,0 & 12,0 & \\
\hline & $13 / 11 / 2019$ & 12,6 & 16,4 & \\
\hline & $20 / 11 / 2019$ & 5,3 & 23,7 & Grietas más grandes \\
\hline & $22 / 11 / 2019$ & 2,7 & 26,3 & \\
\hline & $24 / 11 / 2019$ & 0,0 & 29,1 & Valor superó $1 \mathrm{~mm}$ escala de medición (Fin de etapa 1) \\
\hline & $24 / 11 / 2019$ & 28,4 & 29,1 & $\begin{array}{l}\text { Recalibración de extensómetro, poste móvil inclinado } \\
\text { con cabeceo (Inicio de etapa 2) }\end{array}$ \\
\hline & $17 / 12 / 2019$ & 0,0 & 79,6 & $\begin{array}{l}\text { Valor superó 22,1 cm escala de medición (Fin de etapa } \\
\text { 2) }\end{array}$ \\
\hline \multirow{10}{*}{ CENTRAL } & $24 / 10 / 2019$ & 29,2 & 0,0 & Instalación-ensamblaje \\
\hline & $3 / 11 / 2019$ & 29,2 & 0,0 & Lectura después de sismo cercano \\
\hline & $9 / 11 / 2019$ & 29,2 & 0,0 & \\
\hline & $13 / 11 / 2019$ & 29,2 & 0,0 & \\
\hline & $20 / 11 / 2019$ & 29,1 & 0,1 & \\
\hline & $24 / 11 / 2019$ & 29,1 & 0,1 & \\
\hline & $17 / 12 / 2019$ & 28,8 & 0,4 & \\
\hline & $9 / 1 / 2020$ & 28,2 & 1,0 & \\
\hline & $19 / 01 / 2020$ & 28,3 & 0,9 & \\
\hline & $10 / 3 / 2020$ & 28,2 & 1,0 & \\
\hline \multirow{11}{*}{ OCCIDENTAL } & $24 / 10 / 2019$ & 29,0 & 0,0 & Instalación-ensamblaje \\
\hline & $3 / 11 / 2019$ & 28,5 & 0,5 & Lectura después de sismo cercano \\
\hline & $9 / 11 / 2019$ & 27,9 & 1,1 & \\
\hline & $13 / 11 / 2019$ & 27,9 & 1,1 & Grietas N-S en pie de escarpe \\
\hline & $20 / 11 / 2019$ & 27,8 & 1,2 & \\
\hline & $22 / 11 / 2019$ & 27,6 & 1,4 & \\
\hline & $24 / 11 / 2019$ & 27,6 & 1,4 & \\
\hline & $17 / 12 / 2019$ & 26,2 & 2,8 & \\
\hline & $9 / 1 / 2020$ & 25,8 & 3,2 & \\
\hline & $19 / 01 / 2020$ & 24,3 & 4,7 & Ceja de medición reajustada en $24,3 \mathrm{~cm} \pm 0,5 \mathrm{~mm}$ \\
\hline & $10 / 3 / 2020$ & 21,0 & 8,0 & \\
\hline
\end{tabular}




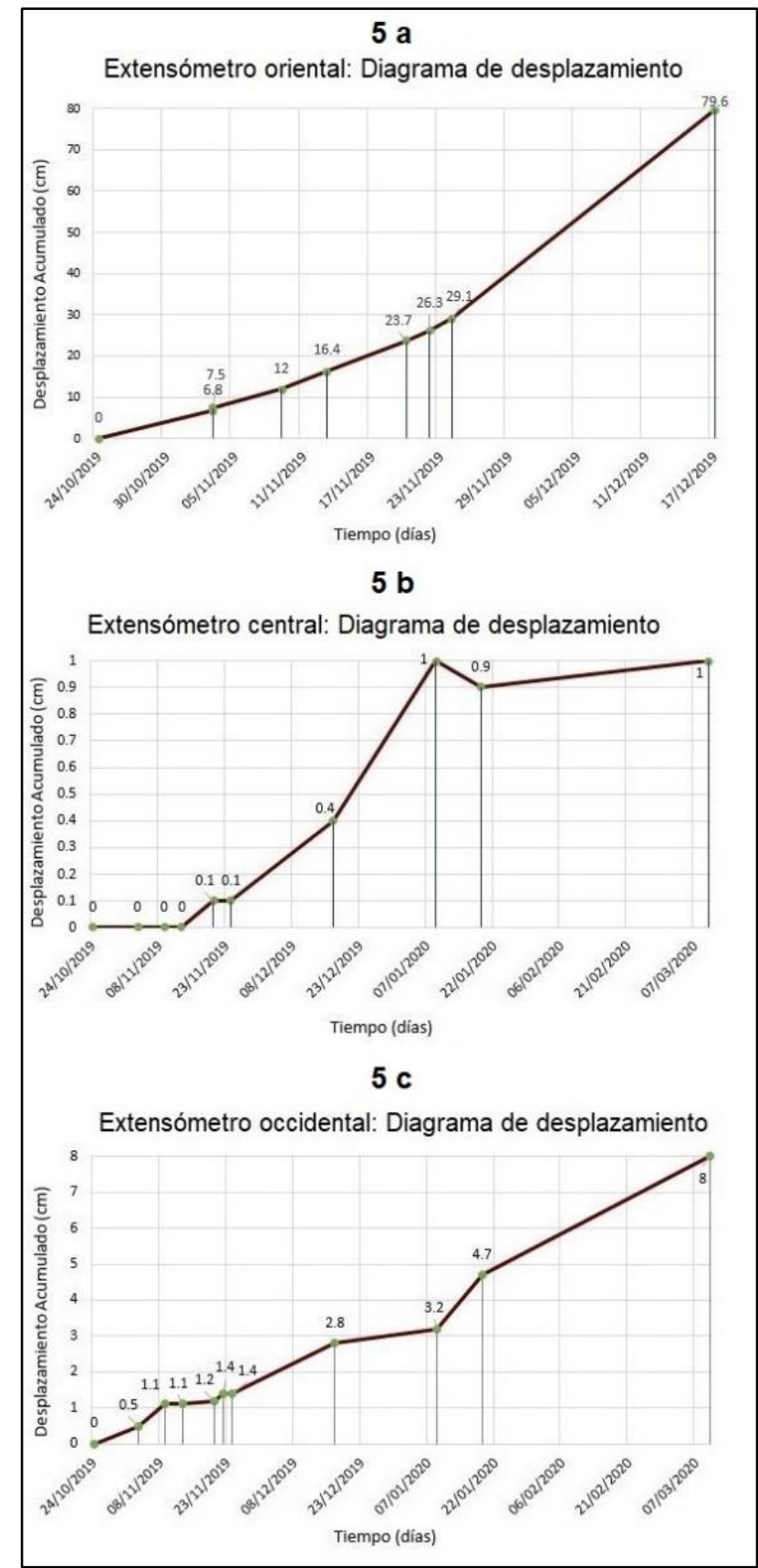

Figura 5. Valores de desplazamientos acumulados en los extensómetros oriental (5a), central (5b) y occidental $(5 \mathrm{c})$. El valor registrado del extensómetro oriental es de dos periodos
Tabla 2. Datos de longitud (UTM X), latitud (UTM Y) y elevación (Altura) registrados con Estación Total en tres campañas de campo

\begin{tabular}{cccc}
\hline \multirow{2}{*}{ FECHA } & \multicolumn{2}{c}{ COORDENADAS UTM } & \begin{tabular}{c} 
ALTURA \\
\cline { 2 - 3 } \\
\cline { 2 - 3 }
\end{tabular} \\
\hline
\end{tabular}

\section{ESTACIÓN CERRO PUNTERAS-MOJÓN 1}

OBSERVACIÓN: Ubicado 100 metros al costado derecho de la vía antes de la Y a Cachi Alto, en una división alta (zanja) de terrenos

\begin{tabular}{rrrr}
\hline $11 / 13 / 2019$ & 750869,609 & 9892288,072 & 3408,319 \\
$12 / 17 / 2019$ & 750870,202 & 9892288,74 & 3408,264 \\
$1 / 9 / 2020$ & 750869,373 & 9892288,514 & 3408,125 \\
\hline \multicolumn{4}{l}{ ESTACIÓN CERRO PUNTERAS-MOJÓN 2 } \\
\hline
\end{tabular}

OBSERVACIONES: Ubicado en la zona del

deslizamiento, en zona boscosa y en una elevación

\begin{tabular}{rrrr}
$11 / 13 / 2019$ & 750758,363 & 9892418,996 & 3364,71 \\
$12 / 17 / 2019$ & 750759,195 & 9892419,371 & 3364,665 \\
$1 / 9 / 2020$ & 750759,86 & 9892419,956 & 3364,417 \\
\hline
\end{tabular}

ESTACIÓN CERRO PUNTERAS-MOJÓN 3

OBSERVACIONES: Ubicado 80 metros, costado derecho

de la vía a Cachi, tras casa de señor Sixto

\begin{tabular}{rrrr}
\hline $11 / 13 / 2019$ & 750536,722 & 9892242,528 & 3431,656 \\
$12 / 17 / 2019$ & 750536,706 & 9892242,511 & 3431,803 \\
$1 / 9 / 2020$ & 750536,53 & 9892242,488 & 3431,643 \\
\hline
\end{tabular}

ESTACIÓN CERRO PUNTERAS-MOJÓN 4

OBSERVACIONES: Ubicado 150 metros al oeste del extensómetro occidental

\begin{tabular}{rrrr}
\hline $11 / 13 / 2019$ & 750373,452 & 9892288,842 & 3460,822 \\
$12 / 17 / 2019$ & 750373,399 & 9892287,54 & 3460,789 \\
$1 / 9 / 2020$ & 750374,561 & 9892287,328 & 3460,721 \\
\hline
\end{tabular}

\section{ESTACIÓN CERRO PUNTERAS-MOJÓN 5}

OBSERVACIONES: Ubicado a 100 metros al noroeste del extensómetro occidental

\begin{tabular}{rrrr}
\hline $11 / 13 / 2019$ & 750249,384 & 9892473,800 & 3493,494 \\
$12 / 17 / 2019$ & 750249,152 & 9892473,682 & 3493,487 \\
$1 / 9 / 2020$ & 750249,072 & 9892473,621 & 3493,485 \\
\hline \multicolumn{4}{c}{ ESTACIÓN CERRO PUNTERAS-MOJÓN 6} \\
\hline
\end{tabular}

OBSERVACIONES: Ubicado a 400 metros al noroeste del extensómetro occidental

\begin{tabular}{rrrr}
\hline $11 / 13 / 2019$ & 749967,984 & 9892643,135 & 3507,719 \\
$12 / 17 / 2019$ & 749967,991 & 9892643,202 & 3507,709 \\
$1 / 9 / 2020$ & 749967,993 & 9892643,206 & 3507,705 \\
\hline
\end{tabular}

Tabla 3. Valores de los desplazamientos de los 6 mojones en los dos periodos de monitoreo. Los valores negativos en $\mathrm{X}$, Y y H indican

\begin{tabular}{crrr}
\multicolumn{4}{c}{ movimientos relativos } \\
\hline \multirow{2}{*}{ MOJÓN } & $\begin{array}{c}\text { DESPLAZA_ } \\
\text { MIENTO X } \\
(\mathbf{m})\end{array}$ & $\begin{array}{c}\text { DESPLAZA_ } \\
\text { MIENTO Y (m) }\end{array}$ & $\begin{array}{c}\text { DESPLAZA_ } \\
\text { MIENTO H (m) }\end{array}$ \\
\hline \multirow{2}{*}{$\mathbf{1}$} & $-0,593$ & $-0,668$ & 0,055 \\
& 0,829 & 0,226 & 0,139 \\
\hline \multirow{2}{*}{$\mathbf{2}$} & $-0,832$ & $-0,375$ & 0,045 \\
& $-0,665$ & $-0,585$ & 0,248 \\
\hline \multirow{2}{*}{$\mathbf{3}$} & 0,016 & 0,017 & $-0,147$ \\
& 0,176 & 0,023 & 0,16 \\
\hline \multirow{2}{*}{$\mathbf{4}$} & 0,053 & 1,302 & 0,033 \\
& $-1,162$ & 0,212 & 0,068 \\
\hline \multirow{2}{*}{$\mathbf{5}$} & 0,232 & 0,118 & 0,007 \\
& 0,08 & 0,061 & 0,002 \\
\hline \multirow{2}{*}{$\mathbf{6}$} & $-0,007$ & $-0,067$ & 0,01 \\
& $-0,002$ & $-0,004$ & 0,004 \\
\hline
\end{tabular}




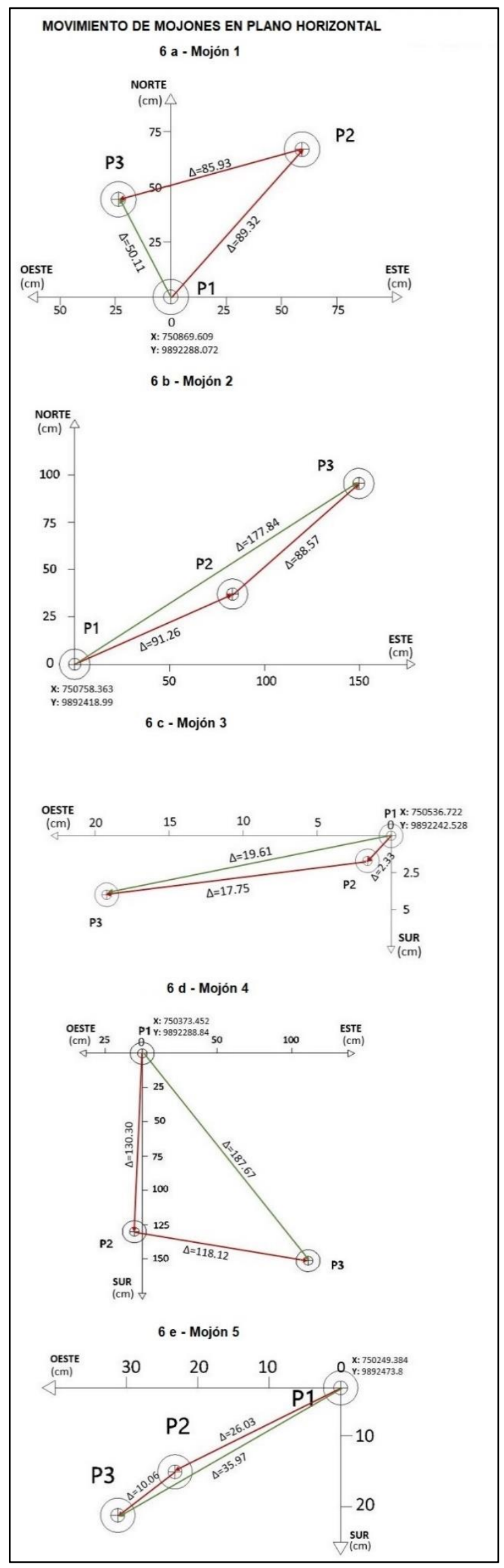

Figura 6. Diagramas mostrando los desplazamientos de los mojones en el plano horizontal

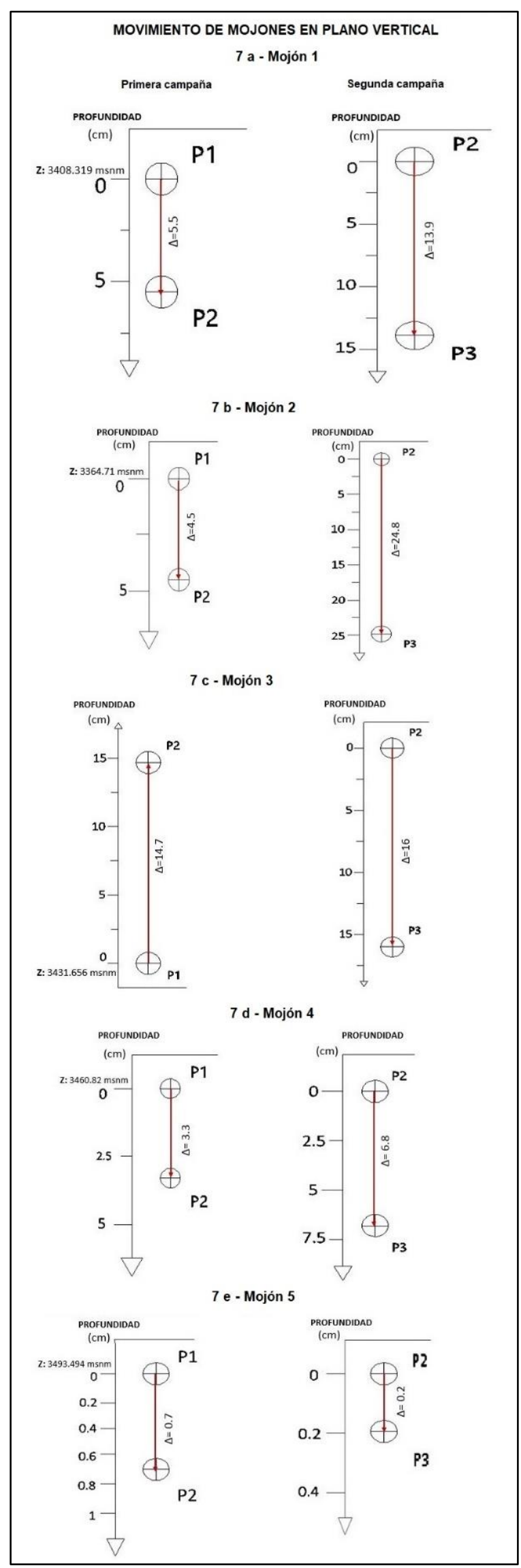

Figura 7. Diagramas mostrando los desplazamientos de los mojones en el plano vertical 
Las deformaciones están localizadas en la zona sur del deslizamiento antiguo de gran magnitud, específicamente en la corona del deslizamiento mencionado, entre los extensómetros central y occidental. Además, otra deformación ocurre en los bordes suroriental y oriental del deslizamiento antiguo. Las deformaciones incluyen principalmente grietas de dimensiones variables. La grieta más occidental tiene \pm 200 metros de longitud, 5 centímetros de abertura y rumbo preferencial NE-SW (Figura 8).

La grieta más oriental tiene más de \pm 350 metros de longitud, \pm 50 centímetros de abertura y rumbo preferencial mayormente NE-SW. Adicionalmente, en la zona norte del deslizamiento antiguo, en la vía antigua a Cachi Alto ocurre un bloque levantado en contrapendiente. La capa superficial del bloque está constituida por cangahua.

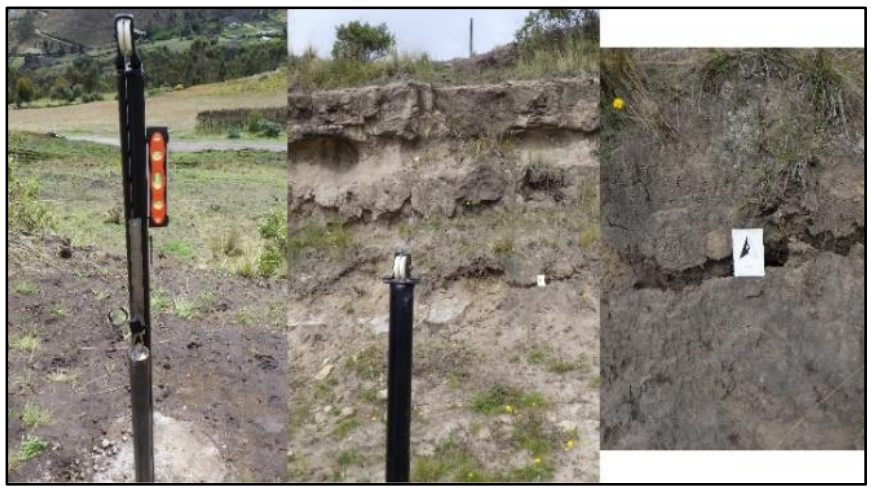

Figura 8. Extensómetro artesanal occidental, a la izquierda Tubo fijo, en el centro Tubo móvil con escarpe antiguo y grieta reciente. La imagen de la derecha incluyendo escala gráfica muestra la grieta de rumbo NE-SW

\section{DISCUSIÓN}

El monitoreo con los extensómetros y la estación total indica que las zonas ubicadas al suroeste, sur y sureste del escarpe principal del deslizamiento antiguo de gran magnitud de Cachi Alto presentan movimientos en los componentes horizontal y vertical. En el componente horizontal los bloques relacionados con los Tubos móviles y los mojones 3, 4 y 5, ubicados en la zona suroccidental tienden a desplazarse al SE, SW y NE, mientras el mojón 1 ubicado en la zona suroriental presenta un desplazamiento al NW (Figura 9). Por otra parte, el Tubo móvil y el mojón 2 ubicados en el cuerpo del deslizamiento antiguo presentan desplazamientos al NE (Figura 9).

Los desplazamientos o movimientos ya mencionados y la ocurrencia de grietas fuertemente inclinadas, recientes y de dimensiones variables en la zona de la corona del deslizamiento antiguo, estructuras paralelas y perpendiculares a los sistemas de fallamiento, así como en varios sectores del cuerpo del deslizamiento antiguo, determinan que toda zona relacionada con la corona del deslizamiento antiguo de Cachi Alto y el cuerpo del mencionado deslizamiento presentan evidentes indicios de movimiento, aunque en base a información cartográfica geológica el deslizamiento antiguo fue catalogado como un deslizamiento inactivo (Burga, 2019). Sin embargo, considerando los datos obtenidos y la clasificación de Cruden y Varnes (1996), tanto la zona de la corona del deslizamiento antiguo, así como la zona relacionada con el cuerpo del deslizamiento son catalogados como movimientos en masa reactivados.

Las evidencias cuantitativas y cualitativas de reactivación sugieren que el área ubicada al S y SW del escape principal o corona del deslizamiento potencialmente es inestable, aunque la caracterización geomecánica de la cangahua determinó que los taludes del escarpe principal constituidos por cangahua, son estables (Analuisa, 2019).

Con respecto a las causas o el origen de la reactivación de estos movimientos en masa que ocurren en la zona de la corona del deslizamiento antiguo de Cachi Alto, aunque en la zona ocurren indicios de neotectonismo (Burga, 2019), este factor como condicionante todavía no es muy concluyente, razón por la cual la reactivación posiblemente está relacionada con la alta inestabilidad del cuerpo del mencionado deslizamiento.

Considerando el rumbo de las grietas paralelas a los escarpes y la tendencia de los procesos, el origen más probable de los movimientos en masa de la corona del deslizamiento antiguo está condicionado por las estructuras geológicas asociadas con actividad neotectónica, como planos de fallas geológicas o grietas reportadas por Burga (2019). Esto en razón que las deformaciones recientes o indicios de inestabilidad coinciden con deformaciones superficiales antiguas (escarpes de movimientos en masa antiguos).

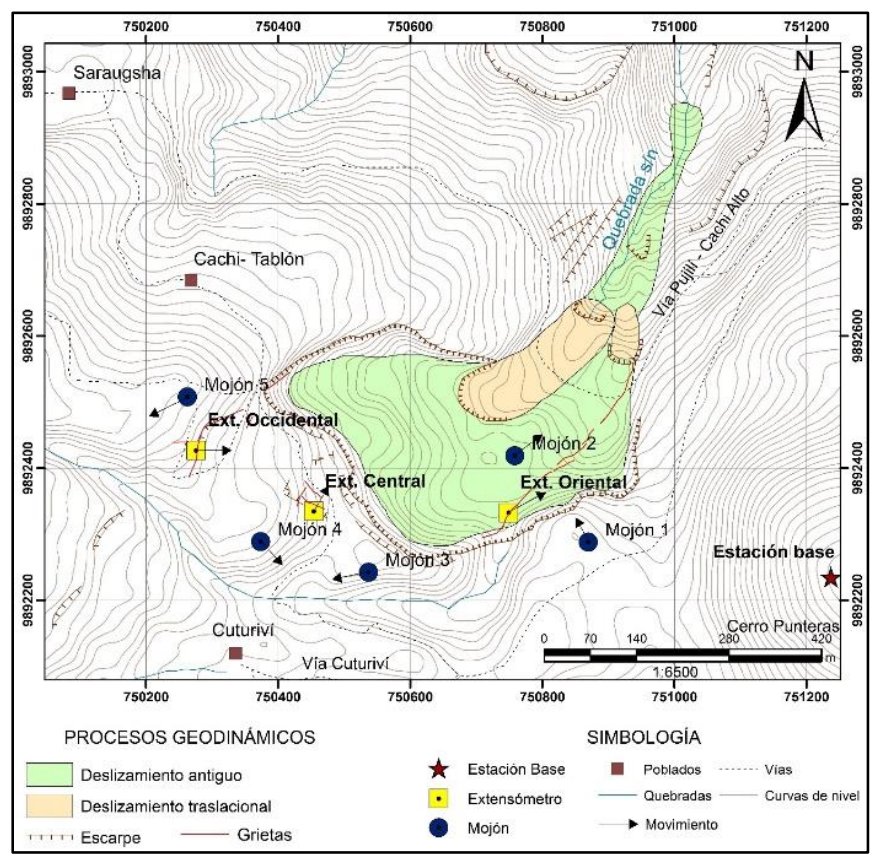

Figura 9. Dirección de desplazamiento o tendencia del movimiento de los mojones utilizados en el monitoreo con Estación Total. Los mojones que se encuentran en la zona sur del deslizamiento antiguo- corona- presentan movimientos con tendencia principalmente al sur. Los mojones 1 y 2 presentan movimientos con tendencia al norte

En este contexto, la hipótesis planteada es que la reactivación del movimiento en masa de Cachi Alto tiene relación con la deformación superficial asociada con un proceso geológico denominado Deformación Gravitacional Profunda en Laderas, 
aspecto sugerido también por Costa C. (Comunicación personal). Estos procesos geológicos presentan rasgos de deformación sin desarrollo de superficie de rotura definidas y baja magnitud de velocidad y desplazamiento (PMA:GCA, 2007), características que se presentan especialmente en la zona relacionada con la corona del deslizamiento antiguo de Cachi Alto. Deformaciones gravitacionales profundas de laderas asociadas con fallamiento activo han sido registrados en muchas regiones montañosas, incluido en los Andes (Audemard, et al., 2010 y Boivin, 2020), aunque en Ecuador la información sobre este tema es escasa.

\section{CONCLUSIONES}

Los movimientos tanto horizontales como verticales determinados con el monitoreo instrumental, y las deformaciones superficiales (grietas y escarpes) determinan que toda la zona ubicada al sur del deslizamiento antiguo de Cachi Alto, conocida como corona de deslizamiento, forma parte de un movimiento en masa catalogado como reactivado. Los movimientos y las deformaciones se formaron durante el último trimestre del 2019 y el primer trimestre del año 2020. La zona reactivada alcanza un área de $\pm 0,1 \mathrm{~km}^{2}$.

Las magnitudes de los movimientos, son muy variables, sugiriendo entonces que el comportamiento dinámico de los materiales es muy heterogéneo. Además, considerando que el talud general esta inclinado al E, la tendencia de los desplazamientos es muy variable y en razón que los bloques se mueven al N, E y S, concluyendo que los movimientos no son compatibles o no tienen relación con los típicos movimientos en masa catalogados como deslizamientos.

Las características mencionadas sugieren que las deformaciones superficiales están relacionadas con procesos de reacomodamiento de bloques en una zona afectada por deformación gravitacional profunda de laderas. Por tal razón es recomendable continuar con el monitoreo usando el mismo instrumental y mediante la aplicación de otras técnicas (geodésicas usando GPS diferencial o Sensores Remotos usando Ortofotos), aprovechando incluso los artefactos ya instalados (mojones metálicos).

El monitoreo debe complementarse con la implementación de un pluviómetro para determinar si las reactivaciones coinciden con periodos de alta pluviosidad, en razón que la estación pluviométrica más cercana se encuentra en el centro del valle Interandino en el Aeropuerto Cotopaxi a $\pm 15,7 \mathrm{~km}$ al ENE.

Finalmente, a partir de los resultados obtenidos de los movimientos en masa de Cachi Alto, Pujilí, Ecuador, se concluye que con una baja inversión económica ( \pm USD 3000 considerando la construcción de los componentes, trabajo de campo para socialización, instalación y registro de datos) se puede obtener resultados confiables, los cuales son útiles para la toma de decisiones oportunas, que permiten precautelar la seguridad de la comunidad. La confiabilidad está basada en la comparación entre los datos de los instrumentos, así como con las deformaciones superficiales cartografiadas, y la seguridad que los instrumentos no fueron alterados o movidos por causas antrópicas (acción de los animales).

\section{AGRADECIMIENTOS}

Los autores agradecemos a la Universidad Central, en especial a la Facultad de Ingeniería en Geología, Minas, Petróleos y Ambiental, así como a la Dirección de Investigación por los fondos aportados y las facilidades prestadas. Por otra parte, agradecemos al Ing. Elías Ibadango por su aporte y la traducción al inglés, así como también a los estudiantes de la asignatura de Geología de Campo (periodo 2019-2020) de la FIGEMPA que apoyaron en el trabajo de campo y en el registro de datos, en especial a Pamela Acosta y Edison Caizaluisa. Finalmente, agradecemos al Gobierno Descentralizado del Cantón Pujilí, en especial a Evelyn Otañez por la colaboración y apoyo en la organización del trabajo de campo.

\section{REFERENCIAS}

Analuisa, E. (2019). Estabilización de laderas en la zona del deslizamiento de Cachi, ubicado en la parroquia y cantón Pujilí, provincia de Cotopaxi. Trabajo teórico de titulación previo a la obtención del título de Ingeniero de Minas. Universidad Central del Ecuador, Quito.

Audemard, F. A., Beck, C. \& Carrillo, E. (2010). Deep-seated gravitational slope deformations along the active Boconó Fault in the central portion of the Mérida Andes, western Venezuela. Geomorpholgy, 124 (3/4), 164-177. Elsevier.

Boland, M., Pilatasig, L., Ibadango, E., McCourt, W., Aspden, J., Hughes, R., \& Beate, B. (2000). Mapa Geológico de la Cordillera Occidental entre $0^{\circ}$ and $1^{\circ} \mathrm{N}$. Proyecto de Desarrollo Minero y Control Ambiental, Programa de Información Cartográfica y Geológica. CODIGEM-BGS. Quito.

Burga, S. (2019). Caracterización Litológica - Estructural y Evaluación de los Deslizamientos en la zona de Cachi, Pujilí, Provincia de Cotopaxi. Trabajo de Titulación, modalidad proyecto de investigación previo a la obtención del Título de Ingeniero en Geología. Universidad Central del Ecuador. Quito.

Cruden, D.M. \& Varnes, D.J. (1996). Landslide types and processes. In: Landslides, Investigation and Mitigation. Special Report 247, Transportation Research Board, Washington, 36-75.

Hughes, R., Bermúdez, R. y Espinel, G. (1998). Mapa Geológico de la Cordillera Occidental del Ecuador entre $0^{\circ}$ y $1^{\circ} \mathrm{S}$. Proyecto de Desarrollo Minero y Control Ambiental. Servicio Geológico Británico-Ministerio de Energía y Minas. Quito - Ecuador.

Hutchinson, J.N. (1988). Morphological and geotechnical parameters of landslide in relation to geology and hydrogeology, en Memorias, $5^{\text {th }}$ International Conference on Landslides, Lausanne, p. 3-35.

León, E. Oleas, M. Pilatasig, L. (2007). Monitoreo de deslizamientos con extensómetros de fabricación casera de bajo costo. Caso de estudio Deslizamiento Tumba-San Francisco, Provincia de Chimborazo, País Ecuador. Gestión de Riesgos por deslizamientos. Editor Guardado, R. GEDES 2007.

Mc Court, W. J., Duque, P. \& Pilatasig, L. (1997). Mapa Geológico de la Cordillera Occidental entre $1^{\circ} S$ y $2^{\circ}$ S. Proyecto de Desarrollo Minero y Control Ambiental, Programa de Información Cartográfica y Geológica. CODIGEM-BGS. Quito.

Pérez, A. (2019). Informe del Proceso de Preparación, Adecuación y Ensamblaje del Prototipo del Extensómetro Artesanal y Mojón Metálico. Proyecto Evaluación del Estado de Actividad de los Movimientos en Masa, usando Extensómetros Artesanales y Estación Total, Caso de Estudio Cachi Alto-Pujilí. Dirección de 
Investigación. Universidad Central del Ecuador. Informe no publicado.

Pilatasig, L., Ibadango, E., Bustillos, J., Ruiz, G., Troncoso, L., Mateus, A. \& Alban, G. (2019). Evaluación de Movimientos en Masa y Relación con la Litoestratigrafía y el Tectonismo, Cachi Alto-Pujilí. Universidad Central del Ecuador, Facultad de Ingeniería en Geología, Minas, Petróleos y Ambiental. Programa de Vinculación con la Sociedad UCE. Informe no publicado.

Proyecto Multinacional Andino: Geociencias para la Comunidades Andinas. (2007). Movimientos en Masa en la Región Andina: Una Guía para la Evaluación de Amenazas. Servicio Nacional de Geología y Minería, Publicación Geológica Multinacional, No 4, 432 p., 1 CD -ROM.

Riemer, W. Locher, T. \& Nuñez, I. (1988). Mechanics of deep seated mass movements in metamorphic rocks of the Ecuadorian Andes. Landslides. Proceedings of the Fifth International Symposium on Landslides. Bonnard, C. Editor. A.A.Balkema / R o t i e r d a m / Brookfield / 1988 .

Referencias electrónicas

Boivin, C. (2020). Long and short time evolution of deep seated gravitational slope deformation: contribution to knowledge of phenomena for the management of alea in the Alpine mountains. Université de Strasbour Université de Strasbourg, Institut de physique du globe de Strasbourg (IPGS), Déformation Active, France. Obtenido de: https://doi.org/10.5194/egusphere-egu2020-4890.( Octubre, 2020).

Teshebaeva, K., Echtler, H., Bookhagen, B., Strecker, M. R. (2019): Deepseated gravitational slope deformation (DSGSD) and slow moving landslides in the southern Tien Shan Mountains: new insights from InSAR, tectonic, and geomorphic analysis. Earth Surface Processes and Landforms, 44, 12, pp. 2333-2348. Obtenido de: DOI: http://doi.org/10.1002/esp.4648.

\section{BIOGRAFÍAS}

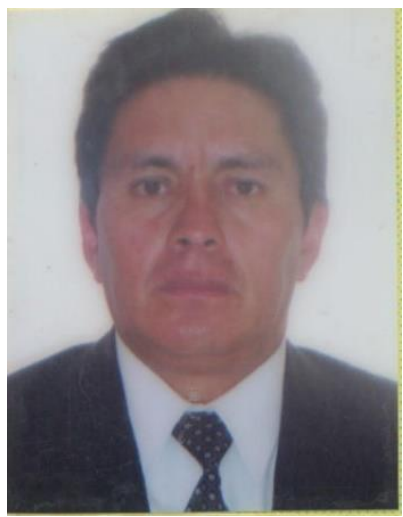

Luis Felipe Pilatasig Moreno, nacido el 7 de noviembre de 1964 en la ciudad de Latacunga, provincia de Cotopaxi. Estudió en la Universidad Central del Ecuador donde obtuvo el título de Ingeniero en Geología. Realizó estudios de posgrado en la Universidad de Exeter de Gran Bretaña. Ejerció funciones de Técnico y Director en el Ministerio de Energía y MinasMinisterio de Recursos Naturales No Renovables, e Instituto Nacional de Investigación Geológico Minero Metalúrgico por 20 años. Docente Universitario por más de 7 años. Actualmente Docente-Investigador de la Facultad de Ingeniería en Geología, Minas, Petróleos y Ambiental (FIGEMPA) de la Universidad Central del Ecuador.

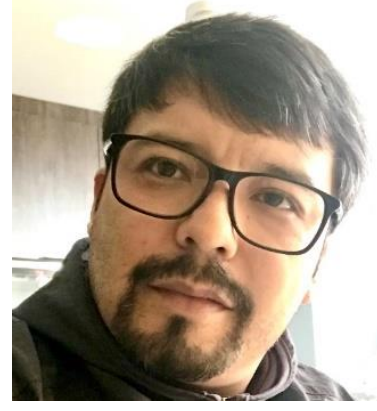

Jorge Eduardo Bustillos Arequipa, nacido el 15 de abril de 1981 en el Cantón Saquisilí de la Provincia de Cotopaxi. Estudió en la Escuela Politécnica Nacional obteniendo el título de Ingeniero Geólogo. Estudios de posgrado en la Universidad Sophia Antipolis de NiceFrancia y la Universidad de Ginebra en Suiza. Sismólogo y Vulcanólogo del IGEPN por 10 años, Docente Universitario por más de 13 años. Director de la Carrera de Ingeniería en Geología de la Universidad Central del Ecuador (UCE) durante 5 años. Actualmente Docente-Investigador de la Facultad de Ingeniería en Geología, Minas, Petróleos y Ambiental (FIGEMPA) de la UCE.

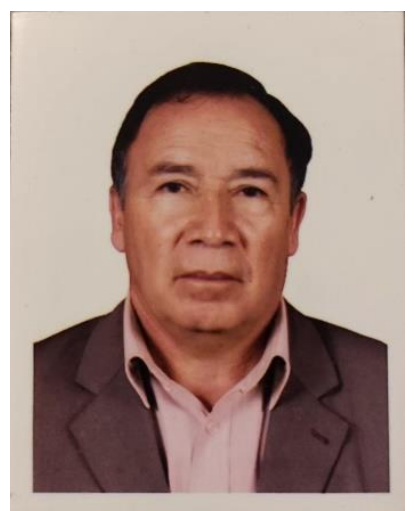

Luis Fabián Jácome Calderón, nacido el 6 de junio de 1951 en la ciudad de Atuntaqui, provincia de Imbabura. Estudió en la Universidad Central del Ecuador donde obtuvo el título de Ingeniero en Minas. Realizó estudios de posgrado en la Universidad Central del Ecuador-Universidad de Huelva de España. Ejerció funciones técnicas en la Corporación Nacional del Cobre de Chile, CODELCOCHILE, MINERA ANDINA, Instituto Ecuatoriano de Recursos Hidráulicos INERHI-Dpto. de Geotecnia, AGROMAN, Instituto Ecuatoriano de Minería-INEMIN, Dirección Nacional de Minería-MEM y Agencia de Regulación y Control Minera. Es Docente Universitario de la Facultad de Ingeniería en Geología, Minas, Petróleos y Ambiental (FIGEMPA) de la Universidad Central del Ecuador.

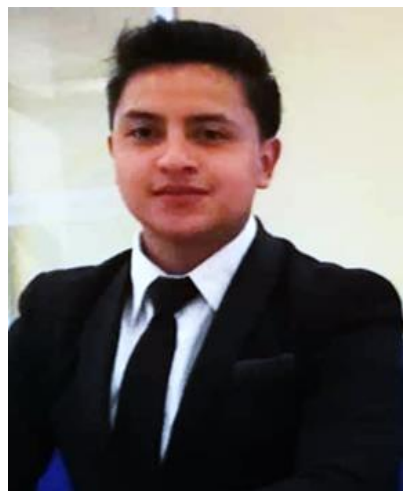

Alexander David Mariño Moyón, oriundo de la ciudad de Guaranda. Se graduó de Ingeniero en Geología de la Universidad Central del Ecuador en 2021. En 2018 participó en el proyecto "Evaluación geológicaestructural y caracterización del deslizamiento de CachiSanta Bárbara". Se desempeñó como geólogo de exploración en el proyecto minero Fruta del Norte en 2019. En su trayecto académico se destacó como miembro de la Asociación de Estudiantes y AAPG STUDENT 
CHAPTER-SEDE UCE, promoviendo eventos educativos y capacitaciones; destacando habilidades de liderazgo; participó en numerosos congresos orientados a cartografía y exploración geológica. 